\title{
Avaliação de coagulação, fibrinólise e proteína C em pacientes de risco e com doenças coronarianas
}

\author{
Evaluation of coagulation, fibrinolysis and protein $\mathrm{C}$ in risk patients and patients presenting coronarian diseases
}

\author{
Christiane Vieira Reis ${ }^{1}$ \\ Lauro Mello Vieira² \\ Luci Maria Sant'Anna Dusse ${ }^{3}$ \\ Eduardo Ferraz Coelho ${ }^{1}$ \\ Milton Luiz de Freitas ${ }^{3}$ \\ Márcio Ribeiro Diniz ${ }^{3}$ \\ Cyntia do Carmo Costa ${ }^{3}$ \\ Maria das Graças Carvalho ${ }^{2}$
}

\begin{tabular}{|c|c|}
\hline ermo & 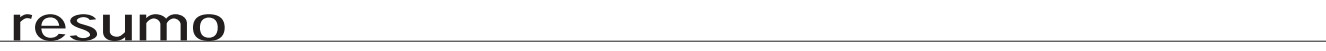 \\
\hline $\begin{array}{l}\text { Doenças coronarianas } \\
\text { Cascata da coagulação } \\
\text { Anticoagulação natural } \\
\text { Fibrinólise } \\
\text { Estado trombótico }\end{array}$ & $\begin{array}{l}\text { A hemostasia é resultante do equilíbrio entre pró-coagulantes e anticoagulantes, envolvendo vasos, } \\
\text { plaquetas, proteínas da coagulação e da fibrinólise e anticoagulantes naturais. Todos estes componentes } \\
\text { estão inter-relacionados, constituindo os sistemas de coagulação, anticoagulação e fibrinólise. Muitos } \\
\text { fatores, genéticos ou adquiridos, podem contribuir para romper este equilíbrio, levando a estados de } \\
\text { hipo ou hipercoagulabilidade. Em doenças coronarianas como a angina e o infarto, há uma maior } \\
\text { ativação das plaquetas e das proteínas da coagulação, favorecendo a formação de trombos. Na tentativa } \\
\text { de restaurar a hemostasia, ocorre a intervenção do sistema fibrinolítico, o qual promove a lise do coágulo } \\
\text { e desobstrui o vaso. Neste trabalho foram avaliados os mecanismos da coagulação e da fibrinólise } \\
\text { e a proteína C, um anticoagulante natural. Foram estudados } 20 \text { pacientes com doenças coronarianas, } \\
\text { notadamente angina de peito ( } n=8 \text { ) e infarto agudo do miocárdio ( } n=12 \text { ), além de pacientes } \\
\text { potencialmente em risco de desenvolver doença cardiovascular ( } n=17) \text {. O grupo infarto foi pareado } \\
\text { com indivíduos sadios do ponto de vista clinicolaboratorial (grupo-controle, } n=12 \text { ). Os resultados } \\
\text { revelaram uma diferença significativa nos níveis de fibrinogênio nos grupos de angina e infarto quando } \\
\text { comparados ao grupo-controle. Níveis de proteína C ativada também mostraram diferença significativa } \\
\text { entre os grupos de risco e infarto. Os demais parâmetros hemostáticos avaliados não diferiram significati- } \\
\text { vamente entre os grupos estudados, porém foi observada uma tendência à hipercoagulabilidade nos } \\
\text { grupos de pacientes quando comparados ao grupo-controle. }\end{array}$ \\
\hline
\end{tabular}

abstract

Hemostasia consists of a balance between procoagulants and anticoagulants involving vessels, platelets, clot and fibrinolysis proteins and natural anticoagulants. All the components are interrelated constituting the coagulation, anticoagulation and fibrinolysis systems. Many genetic or acquired factors may contribute to break this balance leading to hypo or hypercoagulability states. In coronarian diseases, such as angina pectoris and acute myocardial infarction, an exacerbated activation of the platelets and clot proteins occurs, favouring thrombus formation. In order to restore hemostasia, the fibrinolytic system intervention occurs, what promotes the clot lysis removing obstruction from the vessels. In this work, the clot and fibrinolysis systems and protein C, a natural anticoagulant, were evaluated. Twenty patients with coronarian diseases including angina pectoris $(n=8)$ and acute myocardial infarction $(n=12)$ were studied, besides patients showing potential risk for developing cardiovascular disease $(n=17)$. The group of infarcted patients was matched to healthy subjects under the clinical and laboratory points of view (control group, $n=12$ ). The results for angina and infarction groups revealed a significant difference in fibrinogen plasma levels compared to control group. Activated protein C plasma levels also showed a significant difference between the risk and infarction groups. The other hemostatic evaluated parameters did not differ significantly between the studied groups. However, a tendency to hypercoagulability was observed in the patient groups compared to the control group. key words

Coronarian diseases

Coagulation cascade

Natural anticoagulation

Fibrinolysis

Thrombotic state 


\section{Introdução}

A hemostasia é resultante do equilíbrio entre proteínas pró-coagulantes e anticoagulantes, na qual estão envolvidos os vasos, as plaquetas, as proteínas da coagulação e da fibrinólise e os anticoagulantes naturais. Todos estes componentes estão inter-relacionados, constituindo os sistemas da coagulação, da anticoagulação e da fibrinólise (7). Muitos fatores, genéticos ou adquiridos, podem contribuir para romper este equilíbrio, levando a estados de hipo ou hipercoagulabilidade. Como manifestação de um estado de hipercoagulabilidade, a trombose constitui um problema clínico de considerável gravidade e morte ocasional $(2,6)$.

Evidências têm mostrado que a trombose pode contribuir para a ocorrência da doença cardíaca coronariana, não somente como uma complicação aguda do ateroma e da placa rompida, mas também como uma causa contínua do processo crônico da aterogênese (9).

A trombose da artéria coronariana ocorre principalmente em um local acometido por lesão aterosclerótica e resulta em uma situação clínica denominada de infarto agudo do miocárdio (IAM). Considerando as características patológicas aterotrombóticas do IAM, é razoável especular que os efeitos dos fatores de risco trombóticos interagem sinergicamente com os fatores de risco ateroscleróticos, aumentando a sua ocorrência (10).

São muitos os fatores de risco relacionados com o infarto, entre eles a idade e o sexo, sendo rara a sua incidência em pessoas com menos de 45 anos de idade e em mulheres jovens. Outros fatores de risco como o tabagismo, a obesidade, a hipercolesterolemia, a hipertensão e o diabetes contribuem para o desenvolvimento do IAM (10). Fatores de risco trombótico como o aumento dos níveis plasmáticos de fibrinogênio, fator VII, inibidor do ativador do plasminogênio tipo 1 (PAl-1) e outros também estão relacionados com a incidência de doença coronariana (4).

Sabendo-se que a presença de fatores de risco pode resultar na ativação da coagulação e que o infarto é, em geral, conseqüente a um evento trombótico, o presente trabalho tem como principal hipótese de estudo a premissa de que há uma relação direta entre o agravamento da condição clínica e a alteração dos mecanismos da coagulação e da fibrinólise. Desta forma, a determinação de um perfil hemostático de risco para discriminar pacientes que ainda não apresentaram o IAM pode constituir uma ferramenta de grande valor para o estabelecimento de medidas profiláticas que poderão reverter em benefícios a indivíduos ainda assintomáticos. Além disso, a definição do referido perfil poderá ter importância no acompanhamento de pacientes que já desenvolveram IAM e que estão submetidos a tratamento, ou seja, os níveis plasmáticos dos marcadores de hipercoagulabilidade poderão orientar o clínico quanto ao sucesso ou fracasso do esquema terapêutico adotado.

Finalmente, cumpre ressaltar que o achado de testes laboratoriais cujos resultados já se mostram alterados em pacientes de risco, porém ainda assintomáticos, seria de grande valor, considerando que quanto mais precocemente forem determinadas as alterações que favorecem a formação de trombos, mais rapidamente medidas profiláticas poderão ser adotadas. Desta forma, se a hipótese principal de trabalho for verdadeira, é de se esperar que a detecção precoce de alterações nos mecanismos da coagulação e fibrinólise em pacientes que ainda não apresentaram a forma grave de doença cardiovascular seja extremamente importante para o estabelecimento de um perfil hemostático de risco para o desenvolvimento desta doença.

Os objetivos deste trabalho foram investigar, por meio de alguns marcadores específicos, se a cascata da coagulação e a fibrinólise estão ativadas em pacientes com doença coronariana e com fatores de risco, além de investigar a possível existência de algum marcador precoce de hipercoagulabilidade nestes pacientes.

\section{Material e métodos}

Foi avaliado um total de 49 pacientes, compreendendo:

a) grupo de 12 pacientes com infarto agudo do miocárdio que apresentaram o quadro clínico de formigamento e dor anginosa típica com duração superior a $30 \mathrm{mi}$ nutos, sugestivo de infarto agudo do miocárdio com alterações eletrocardiográficas características, acompanhadas da presença de ondas q patológicas, acrescidas de níveis séricos aumentados da enzima creatinacinase (CK) e sua fração MB (método enzimático com leitura em Cobas Mira). Foram excluídos os pacientes com estas características que faziam uso de aspirina ou de outro antiagregante plaquetário, anticoagulantes (warfarina sódica ou heparina) ou que tinham recebido terapêutica trombolítica antes da colheita da amostra para os testes de coagulação. As amostras de sangue foram colhidas imediatamente antes do início da terapêutica específica; 
b) grupo de oito pacientes que apresentaram sintomatologia clínica de angina de peito. Estes pacientes foram selecionados após realização de eletrocardiograma e dosagem das enzimas cardíacas (CK e CK-MB). Todos os pacientes apresentaram o quadro clínico de dor típica e níveis normais das enzimas cardíacas, tendo sido os mesmos puncionados imediatamente antes de qualquer medida terapêutica;

c) grupo de 17 pacientes que apresentaram dois ou mais fatores de risco como hipertensão arterial (pressão sistólica $\geq 140 \mathrm{mmHg}$ e pressão diastólica de $\geq 85 \mathrm{mmHg}$ ), hipercolesterolemia (níveis de HDL-colesterol inferiores a $45 \mathrm{mg} / \mathrm{dl}$ e níveis de LDL-colesterol superiores a $130 \mathrm{mg} / \mathrm{dl}$ ), obesidade (índice de massa corporal $>30$ ) e tabagismo (mais de dez cigarros por dia). Estes pacientes foram selecionados através do Programa de Prevenção à Aterosclerose do Hospital Socor de Belo Horizonte e compareceram ao laboratório para coleta da amostra de sangue em jejum de 12 horas;

d) grupo de 12 indivíduos sem fatores de risco (grupocontrole), isto é, indivíduos sadios sob o ponto de vista clinicolaboratorial com idade e sexo semelhantes aos dos pacientes infartados. Estes pacientes foram selecionados da comunidade em geral após triagem clinicolaboratorial para excluir qualquer fator de risco, tendo estes comparecido ao laboratório, em jejum de 12 horas, para a coleta da amostra de sangue.

Para a comprovação da ausência de fatores de risco e de doenças nos indivíduos do grupo-controle, foram realizados os seguintes exames laboratoriais: glicose (metodologia enzimática), colesterol total (método enzimáticoTrinder), triglicérides (método enzimático Trinder), HDL-colesterol (sistema de precipitação), fosfatase alcalina (método Roy modificado), creatinina (metodologia utilizando ácido pícrico), uréia (método enzimáticourease), transaminase pirúvica e oxalacética (metodologia de Reitman e Frankel). Para a realização destes testes foram utilizados kits da Labtest e da Analisa. Todos os ensaios foram lidos utilizando-se o espectrofotômetro CELM-E-225 D.

Foram excluídos desse grupo os indivíduos que apresentaram história de doenças renais, endócrinas, hepáticas, auto-imunes, os quais foram triados através de cuidadosa anamnese e dos exames laboratoriais citados acima. Aqueles com idade acima de 65 anos e/ou aqueles que faziam uso de medicamentos que alteravam a coagulação, como antiinflamatórios não-esteroidais, anticoncepcionais, ácido acetilsalicílico (AAS), dipiridamol, ticlopidina e anticoagulantes orais, foram também excluídos.

Os grupos de pacientes com IAM e angina de peito foram selecionados no pronto atendimento (Procordis) do Hospital da Santa Casa de Misericórdia de Belo Horizonte.

A pesquisa foi aprovada pela Comissão de Ética em Pesquisa da Santa Casa. Foi obtido o consentimento livre e esclarecido (pós-informado) de todos os pacientes e informações clínicas de todos os participantes foram coletadas por meio de uma ficha clínica para posterior correlação com dados laboratoriais.

\section{Amostra biológica}

Foram coletadas de cada paciente amostras de $15 \mathrm{ml}$ de sangue venoso, em citrato de sódio, por meio de tubos do sistema Vacutainer (Becton Dickinson). Após a coleta, as amostras foram centrifugadas durante 15 minutos, a 3.000rpm. Após a obtenção das amostras de plasmas, estas foram acondicionadas em tubos Eppendorf, devidamente identificados, e estocadas a uma temperatura de cerca de $-20^{\circ} \mathrm{C}$ até o momento da realização dos testes laboratoriais. Para o grupo-controle e o de risco, além dos $15 \mathrm{ml}$ de sangue em citrato, foram coletadas amostras de sangue em tubo sem anticoagulante $(5 \mathrm{ml})$ para a realização dos exames bioquímicos.

\section{Metodologia}

A determinação dos níveis plasmáticos de PAI-1 foi realizada pelo método Elisa de sanduíche, empregando o kit da Diagnostica Stago (Asserachrom,PAl-1). O fator VII e a proteína $C$ foram determinados por método coagulométrico, utilizando kit da Diagnostica Stago (STA, Deficient VII e Staclot, proteína C, respectivamente), enquanto a determinação dos produtos de degradação da fibrina foi feita de maneira qualitativa e semiquantitativa, utilizando-se um sistema de aglutinação por meio de partículas de látex (FDP Plasma, da Diagnostica Stago). Já o plasminogênio (Stachrom, Plasminogen da Diagnostica Stago) e o fibrinogênio plasmático (Goodwin modificado) (1) foram dosados nas amostras por método colorimétrico.

Para a análise estatística foi empregado o programa Systat, versão 8.03. Os dados foram inicialmente submetidos a uma análise descritiva para a obtenção de médias e desvios padrões. Para testar a existência ou não de diferença significativa entre os grupos para os parâmetros estudados, foi empregada análise de variância (Anova) e, quando se verificou a existência de significância, ou seja, 
$p<0,05$, utilizou-se o teste LSD de Fisher (least significance difference) para testar quais grupos apresentaram diferenças e qual o nível de significância estatística obtido entre elas.

\section{Resultados}

A média e o respectivo desvio padrão de cada parâmetro analisado como o fibrinogênio (Fbr), fator VII (FVII), proteína $C$ ativada (PCA), inibidor do ativador do plasminogênio-1 (PAI-1) e plasminogênio (PIn) em amostras de sangue dos grupos-controle, de risco, com angina e com infarto, podem ser observados na Tabela.

Para as concentrações de fibrinogênio, as médias observadas não apresentaram diferença significativa entre os grupos-controle e de indivíduos com fatores de risco. Já para os pacientes dos grupos de angina e infarto agudo do miocárdio, as médias encontradas diferiram significativamente quando comparadas à média do grupo-controle. Entretanto somente a média do grupo infarto diferiu significativamente quando comparada à do grupo de risco (Figura 1).

Para o fator VII, a média dos resultados obtidos não apresentou diferença significativa entre os grupos. Porém pode ser observada uma tendência à elevação das médias para os grupos de risco, de angina e de infarto quando comparados ao grupo-controle (Figura 2).

Para a proteína $\mathrm{C}$ ativada, somente foi observada diferença significativa entre as médias do grupo de risco e do grupo dos pacientes com infarto. Comparando-se as médias obtidas para o grupo-controle e as médias dos grupos com infarto e com angina, nenhuma diferença significativa foi observada (Figura 3).

Quanto aos níveis plasmáticos do inibidor do ativador do plasminogênio tipo 1 (PAI-1), este não apresentou di-

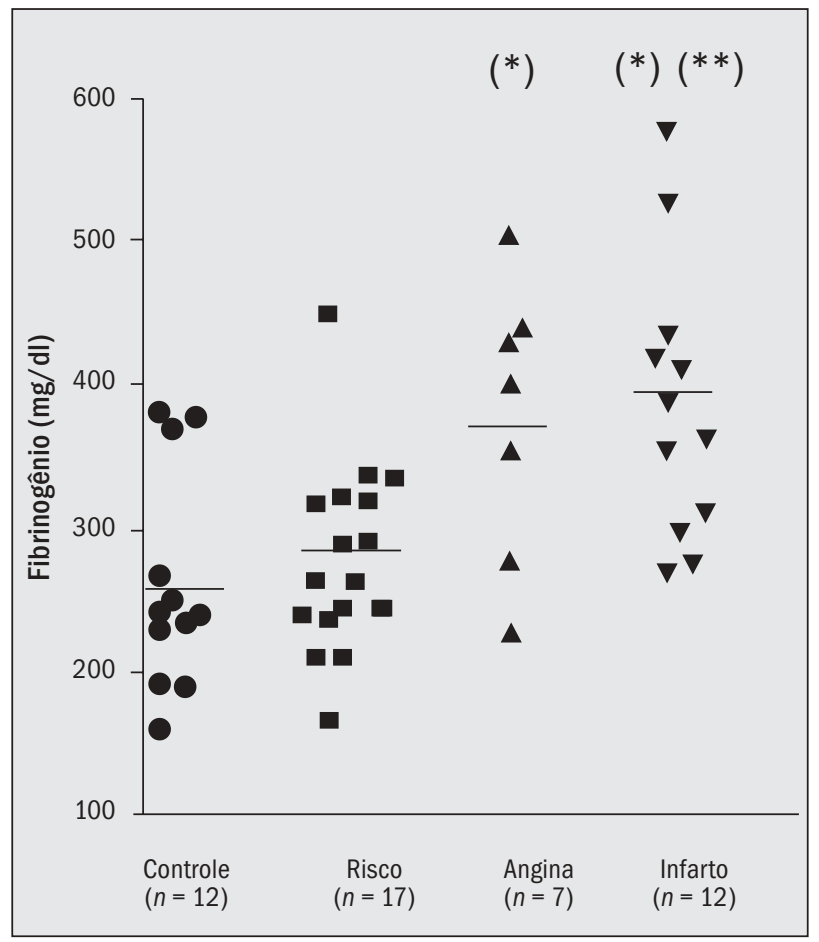

Figura 1 - Resultados das determinações dos níveis de fibrinogênio nos grupos estudados ( $\mathrm{n}=$ número de amostras). As barras horizontais indicam os valores médios de cada grupo. (*) Indica diferença significativa do grupo-controle em relação aos grupos angina e infarto $(p<0,05)$. (*) Indica diferença significativa entre 0 grupo de risco e o grupo infarto $(p<0,05)$

ferença significativa comparando-se as médias dos quatro grupos (Figura 4).

A análise dos resultados obtidos em relação à atividade do plasminogênio demonstrou que não existem diferenças significativas entre os grupos estudados. Apesar de não haver diferença significativa, pode ser observado que as médias dos grupos com angina e com infarto são discretamente inferiores quando comparadas às do grupo-controle (Figura 5).

A Figura 6 mostra os resultados da determinação semiquantitativa dos produtos de degradação da fibrina

\section{Média e desvio padrão para fibrinogênio (Fbr), fator VII (FVII), proteína C ativada (PCA),} Tabela inibidor do ativador do plasminogênio-1 (PAl-1) e plasminogênio (Pln)

\begin{tabular}{lcccc}
\hline Parâmetro & Controle & Risco & Angina & Infarto \\
Fbr $(\mathbf{m g} / \mathbf{d l})(\mathbf{n}=\mathbf{4 8})$ & $261 \pm 75,3(n=12)$ & $279 \pm 65,7(n=17)$ & $374 \pm 96,1^{*}(n=7)$ & $387 \pm 96^{*}(n=12)$ \\
F VII $(\%)(\mathbf{n}=\mathbf{4 9})$ & $85,4 \pm 17,7(n=12)$ & $121 \pm 38,6(n=17)$ & $128 \pm 35,5(n=8)$ & $115 \pm 58,8(n=12)$ \\
PCA $(\%)(\mathbf{n}=\mathbf{4 8})$ & $90,4 \pm 16,7(n=12)$ & $101 \pm 22,7(n=17)$ & $78,4 \pm 23(n=8)$ & $71,4 \pm 21,4^{*}(n=11)$ \\
PAI-1 (ng/ml)(n=49) $28,2 \pm 31,4(n=12)$ & $63,8 \pm 47,4(n=17)$ & $36,3 \pm 30,5(n=8)$ & $69,7 \pm 46,6(n=12)$ \\
Pln $(\%)(\mathbf{n}=\mathbf{4 9})$ & $99 \pm 27(n=12)$ & $107 \pm 22,7(n=17)$ & $89,8 \pm 25,3(n=8)$ & $81,5 \pm 27,6(n=12)$ \\
\hline
\end{tabular}

*Diferenças significativas em relação ao grupo-controle para Fbr e em relação ao grupo de risco para $P C A$, sendo $p<0,05$. 


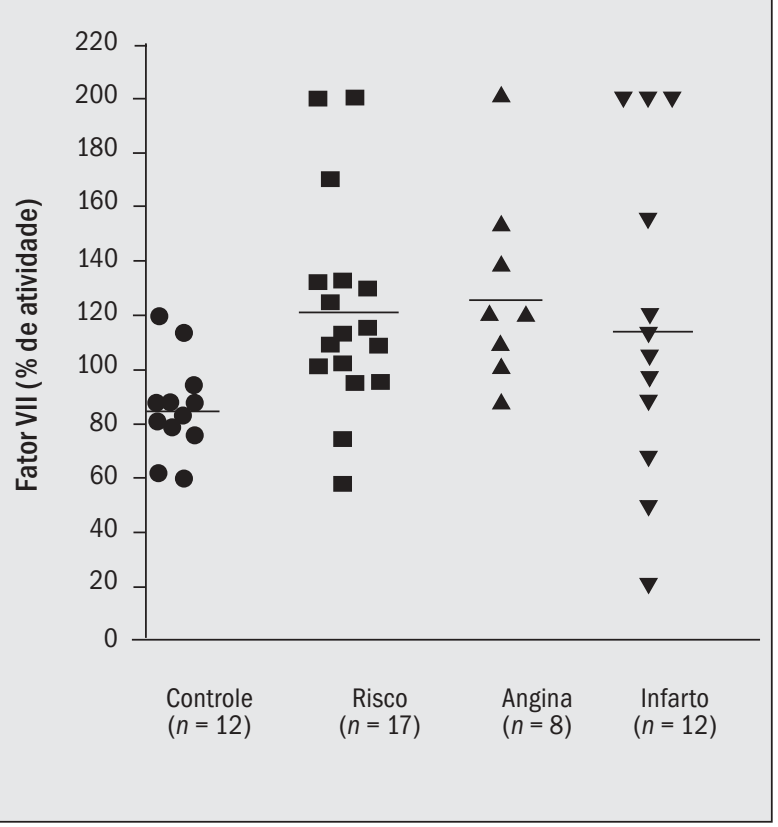

Figura 2 - Resultados da determinação da atividade do fator VII nos quatro grupos estudados ( $\mathrm{n}=$ número de amostras). As barras horizontais indicam os valores médios encontrados para cada grupo. Nenhuma diferença foi encontrada entre as médias dos grupos

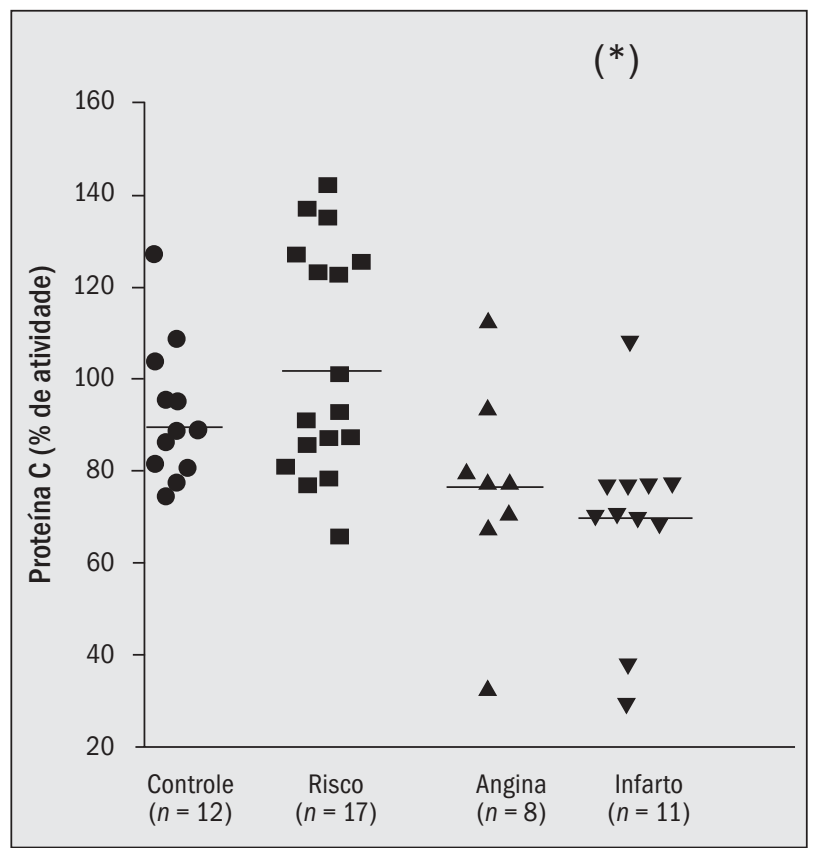

Figura 3 - Resultados das determinações das atividades da proteína $C$ nos grupos estudados ( $\mathrm{n}=$ número de amostras). As barras horizontais indicam os valores médios obtidos para cada grupo. ${ }^{*}$ ) Indica diferença significativa entre o grupo de risco e 0 grupo com infarto $(p<0,05)$

(PDF) nos grupos estudados. Não foi possível realizar a análise estatística devido à igualdade dos resultados fornecidos pelo grupo de risco. No entanto podem ser observados alguns resultados bem mais elevados nos grupos com angina (um caso) e com infarto (dois casos).

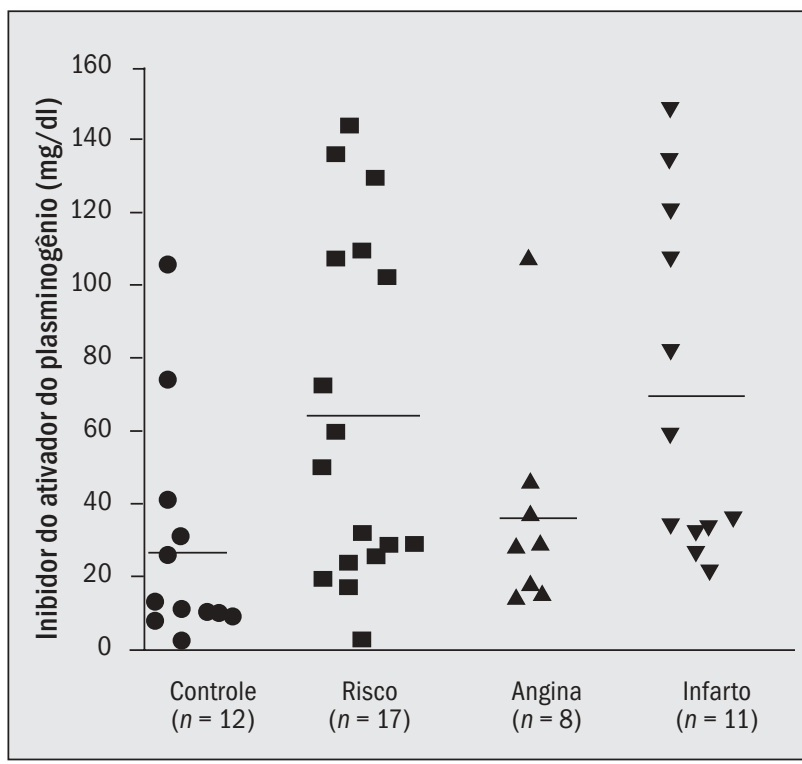

Figura 4 - Resultados dos níveis plasmáticos do inibidor do ativador do plasminogênio tipo 1 nos grupos estudados. As barras horizontais indicam o valor médio encontrado para cada grupo ( $\mathrm{n}=$ número de amostras). Não foi observada diferença entre as médias dos grupos

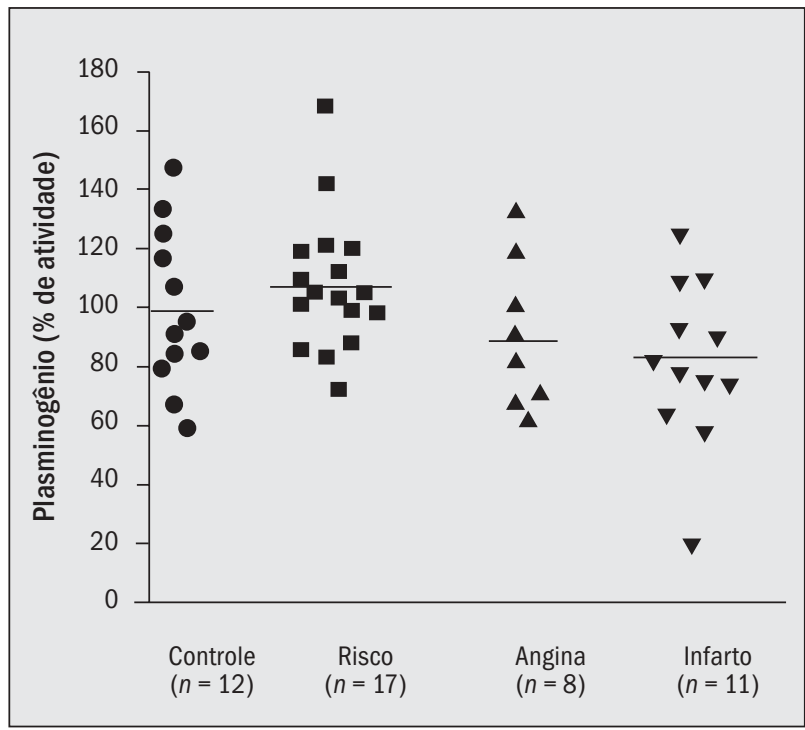

Figura 5 - Resultados da determinação da atividade do plasminogênio nos grupos estudados. As barras horizontais indicam as médias obtidas para cada grupo $(\mathrm{n}=$ número de amostras). Nenhuma diferença foi observada entre as médias dos quatro grupos

\section{Discussão}

Neste estudo foram avaliados parâmetros hemostáticos que investigam o grau de hipercoagulabilidade em situações clínicas nas quais ocorre uma exacerbação da ativação do mecanismo da coagulação e/ou da fibrinólise. A composição dos grupos de pacientes estudados (pacientes com fatores de risco, com angina ou com infarto) foi 


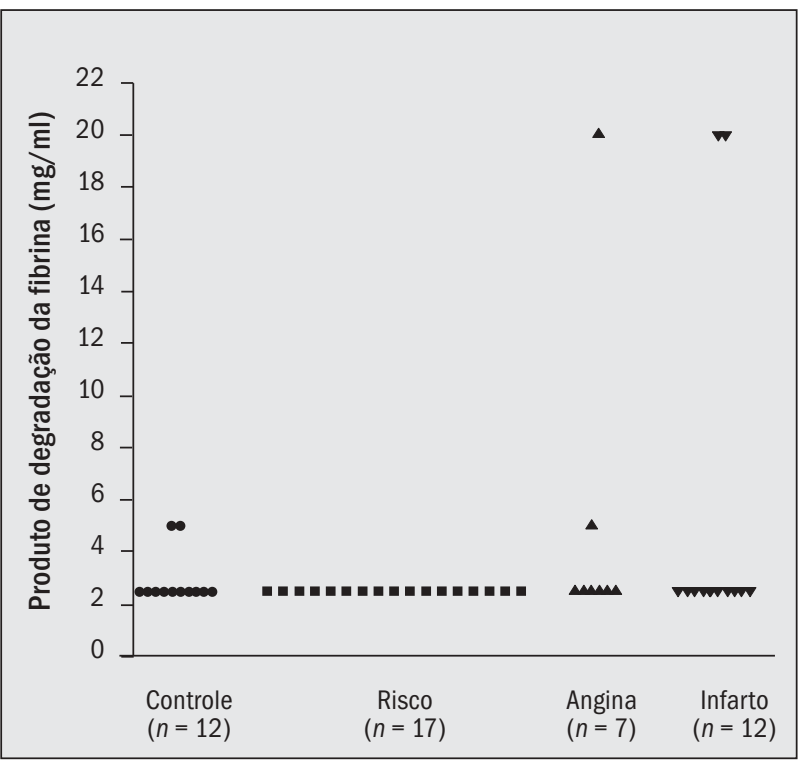

Figura 6 - Resultados da determinação semiquantitativa dos produtos de degradação da fibrina nos grupos de controle, de risco, com angina e com infarto, utilizando a técnica de aglutinação em látex

feita objetivando verificar o grau de ativação do sistema hemostático em uma escala crescente de manifestação de sintomas clínicos. Uma análise global dos dados apresentados na Tabela permite concluir que nas doenças cardiovasculares, notadamente em pacientes com angina ou infarto, há uma tendência a uma maior ativação do mecanismo da coagulação, bem como uma fibrinólise deficiente. Os níveis mais elevados de fibrinogênio, de fator VII e a redução de PCA nesses pacientes, comparando-se com os grupos de controle e de risco, sugerem que o mecanismo da coagulação está ativado, o que predispõe à formação de trombo. Em adição, os níveis elevados de PAl-1 e a baixa disponibilidade de plasminogênio no infarto, em comparação ao grupo-controle, favorecem a manutenção do coágulo de fibrina e, conseqüentemente, o comprometimento do fluxo sangüíneo.

Quanto à avaliação dos níveis plasmáticos de fibrinogênio, o grupo-controle apresentou média significativamente menor que os grupos de pacientes com angina e infarto, enquanto o grupo de risco apresentou diferença significativa apenas em relação ao grupo com infarto. Lowe et al. (8), em 1991, relataram que o aumento dos níveis plasmáticos de fibrinogênio está associado a doença coronariana, o que justifica a elevação dos níveis plasmáticos nos grupos de angina e infarto. Uma análise da Figura 1 permite verificar uma crescente elevação dos níveis plasmáticos dessa proteína de fase aguda e de importância crucial no mecanismo da coagulação nos grupos estudados.
Analisando as médias obtidas para o FVII (Figura 2), pode-se observar uma tendência à elevação da atividade do fator VII nos grupos de risco, angina e infarto, comparando-se com o grupo-controle. Sabe-se que em pacientes obesos, com níveis de triglicérides elevados, a atividade do fator VII aumenta (9). Além disso, os pacientes com angina e IAM podem apresentar fissuras nas lesões ateroscleróticas que expõem o fator tissular. Este, por sua vez, é responsável pela trombogenicidade da placa aterosclerótica através da ativação do fator VII, que é essencial para desencadear a coagulação (3). A discreta redução da atividade do fator VII encontrada no grupo dos infartados pode ser explicada pelo consumo deste fator no processo de formação do trombo intracoronariano encontrado nestes indivíduos.

Com o objetivo de se avaliar o mecanismo da anticoagulação, foi determinada a atividade da proteína C (PC) nos indivíduos estudados (Figura 3). Comparando-se as médias dos grupos de risco, angina e infarto com o grupo-controle, nenhuma diferença foi encontrada, apesar de ser observada uma tendência à ativação da PC nos indivíduos com fatores de risco. Esta discreta ativação da PC poderia ser explicada por um mecanismo de controle para compensar o aumento da coagulação sangüínea encontrada nestes indivíduos. Comparando-se as médias de PC no grupo de risco com o grupo de indivíduos infartados, pode-se observar uma diminuição significativa na média deste último. Este fato pode ser justificado pela lesão endotelial presente nos pacientes com angina e com infarto, o que comprometeria a ativação da PC.

O inibidor do ativador do plasminogênio tipo 1 está relacionado com a regulação do sistema fibrinolítico (7). Em locais onde existem lesões ateroscleróticas, os níveis plasmáticos de PAI-1 estão elevados, o que leva a um desequilíbrio da fibrinólise, favorecendo, assim, a manutenção do trombo (5). Neste trabalho foi verificado que o PAI-1 não diferiu significativamente entre os grupos ( $\mathrm{Fi}$ gura 4). Porém pode-se observar que existe uma tendência à elevação dos níveis deste marcador nos grupos de risco, com angina e com infarto quando comparados ao grupo-controle, o que pode ser um indício de que nestes indivíduos a fibrinólise está prejudicada, predispondo à deposição de trombos nos vasos.

Também com o objetivo de se analisar a fibrinólise, foram avaliados os níveis plasmáticos de plasminogênio (Pln). O Pln, sob ação de ativadores teciduais e plasmáticos, se transforma em plasmina, que é capaz de degradar o coágulo de fibrina (7). Assim, a determinação do plasmi- 
nogênio seria uma maneira indireta de se verificar a eficiência da via da fibrinólise. Neste trabalho, nenhuma diferença foi observada nos grupos, mas uma tendência à diminuição dos níveis de Pln o foi no grupo de pacientes com angina e IAM (Figura 5). Esta diminuição poderia ser explicada pelo consumo do Pln, que seria transformado de maneira a se obter uma quantidade de plasmina capaz de desobstruir o vaso e restaurar a hemostasia nestes indivíduos.

Os produtos de degradação da fibrina não puderam ser analisados devido à homogeneidade dos resultados para o grupo de risco, mas foi observado que para alguns pacientes, principalmente dos grupos angina e infarto, este marcador apresenta-se ativado, o que sugere uma exacerbação na ativação do mecanismo da coagulação nesses indivíduos.

Dessa forma, o presente trabalho permite concluir que a dosagem de fibrinogênio, tradicionalmente empregada para avaliação de risco para doenças coronarianas, apresentou um aumento gradual nos quatro grupos estudados, confirmando os resultados de estudos anteriores que consideraram o fibrinogênio um fator de risco para o desenvolvimento do infarto agudo do miocárdio. Além disso, existe uma tendência à ativação do mecanismo da coagulação e um comprometimento do sistema fibrinolítico nos grupos estudados, o que sugere que, com o agravamento da condição clínica nas doenças coronarianas, haja uma crescente alteração dos mecanismos da coagulação e da fibrinólise.

\section{Agradecimentos}

Agradecemos à Fundação de Amparo à Pesquisa de Minas Gerais (Fapemig) e ao Conselho Nacional de Desenvolvimento Científico e Tecnológico (CNPq) pelo apoio financeiro.

\section{Referências}

1.Carvalho, M.G . \& Silva, M.B.S. Hematologia:técnicas laboratoriais e interpretação. Belo Horizonte: [s. n.], 1988. p. 124.

2. Dahlbäck, B. Inherited thrombophilia: resistance to activated protein $C$ as a pathogenic factor of venous thromboembolism. Blood, 85:607-14, 1995.

3. Falciani, M . et al. Elevated tissue factor and tissue factor pathway inhibitor circulating levels in ischaemic heart disease patients. Thromb. Haemostas., 79:495-9, 1998.

4.Grundy, S.M .et al. Primary prevention of coronary heart disease: guidance from Framingham. Circulation, 9: 1876-87, 1998.

5. $\mathrm{H}$ andt, $\mathrm{S}$. et al. Plasmino gen activator inhibitor-1 secretion by endothelial cells increase fibrinolytic resistance of an in vitro fibrin clot: evidence for a key role of endothelial cells in thro mbolytic resistance. Blood, 87: 4204-13, 1996.

6.Janssen,M.C.H.D -dimer determination to assess regression of deep venous thrombosis. Thromb. Haemostas., 78: 799-802,1997.
7. Jobe, M. I. Mecanisms of coagulation and fibrinolysis. In: Lotspeich-Steininger, C. A . et al. (ed.) Clinical hematology: principles procedures correlations. London: J. B. Lippincott, 1992. p. 579-98.

8. Lowe,G.D.O et al. Relationships of plasma viscosity, coagulation and fibrinolysis to coronary risk factors and angina. Thromb. Haemostas., 65: 339-43, 1991.

9. Scarabin, P.Y. et al.A sso ciations of fibrinogen, factor VII and PA I1 with baseline findings among 10,500 males participants in a prospective study of myocardial infarction. Thromb. Haemostas., 80: 749-56, 1998.

10. Siscovick, D.S. et al. Thrombosis in the young: effects of atherosclerotic risk factors on the risk of myocardial infarction associated with prothrombotic factors. Thromb. Haemostas., 78: 7-12, 1997. 